\title{
LOCAL LIMIT THEOREMS FOR SMOOTHED BERNOULLI AND OTHER CONVOLUTIONS
}

\author{
SERGEY G. BOBKOV ${ }^{1}$ AND ARNAUD MARSIGLIETTI ${ }^{2}$
}

\begin{abstract}
We explore an asymptotic behavior of densities of sums of independent random variables that are convoluted with a small continuous noise.
\end{abstract}

\section{Introduction}

Let $\left(X_{n}\right)_{n \geq 1}$ be independent Bernoulli random variables taking the values \pm 1 with probability $1 / 2$. Given a random variable $X$ with density $p$, let us consider densities $p_{n}$ of the normalized sums

$$
Z_{n}=\frac{1}{\sqrt{n}}\left(X+X_{1}+\cdots+X_{n}\right) .
$$

By the central limit theorem, $Z_{n}$ are convergent weakly in distribution to the standard normal law, which means that, as $n \rightarrow \infty$,

$$
\sup _{a<b}\left|\int_{a}^{b}\left(p_{n}(x)-\varphi(x)\right) d x\right| \rightarrow 0, \quad \text { where } \varphi(x)=\frac{1}{\sqrt{2 \pi}} e^{-x^{2} / 2} .
$$

Therefore, one may wonder whether or not this property can be sharpened as convergence of $p_{n}$ to $\varphi$ in a stronger sense. This question appears naturally in the area of entropic limit theorems with involved problems of estimation of the entropy of $X$, especially in a highdimensional setting (here, we however do not discuss such applications). When $X=0$ and the $X_{k}$ 's are i.i.d., a celebrated result of Gnedenko provides necessary and sufficient conditions for the uniform convergence of $p_{n}$ when these densities exist ([G-K], [B-RR]). Here, we will see that the presence of a non-zero noise $X / \sqrt{n}$ in $Z_{n}$ may enlarge the range of applicability of local limit theorems. Let us focus on the possible convergence in the $L^{2}$-distance

$$
\left\|p_{n}-\varphi\right\|_{2}=\left(\int_{-\infty}^{\infty}\left|p_{n}(x)-\varphi(x)\right|^{2} d x\right)^{1 / 2}
$$

and on the uniform convergence, i.e, for the $L^{\infty}$-norm $\left\|p_{n}-\varphi\right\|_{\infty}$ (which is stronger than the $L^{2}$-convergence). As it turns out, the answers essentially depend on some delicate properties of the density $p$ of $X$, as may be seen from the following characterization in terms of the

2010 Mathematics Subject Classification. Primary 60E, 60F.

Key words and phrases. Central limit theorem, local limit theorem.

1) School of Mathematics, University of Minnesota, Minneapolis, MN 55455 USA. Research was partially supported by NSF grant DMS-1612961.

2) Department of Mathematics, University of Florida, Gainesville, FL 32611 USA. Corresponding author: Arnaud Marsiglietti, Email address: a.marsiglietti@ufl.edu. 
characteristic function

$$
f(t)=\mathbb{E} e^{i t X}=\int_{-\infty}^{\infty} e^{i t x} p(x) d x, \quad t \in \mathbb{R} .
$$

Theorem 1.1. If

$$
\left\|p_{n}-\varphi\right\|_{2} \rightarrow 0 \text { as } n \rightarrow \infty
$$

then

$$
f(\pi k)=0 \quad \text { for all } k \in \mathbb{Z}, k \neq 0 .
$$

Conversely, if $\mathbb{E}|X|<\infty$, and $f^{\prime}$ is square integrable, then the $L^{2}$-convergence (1.1) holds under the condition (1.2).

Under a stronger assumption on $f$, the $L^{2}$-convergence of densities may be strengthened to the uniform convergence.

Theorem 1.2. Assume that the condition (1.2) is fulfilled. If $\mathbb{E}|X|<\infty$, and $f^{\prime}$ is integrable, then the random variables $Z_{n}$ have continuous densities $p_{n}$ such that

$$
\sup _{x}\left|p_{n}(x)-\varphi(x)\right| \rightarrow 0 \quad \text { as } n \rightarrow \infty \text {. }
$$

The square integrability assumption in Theorem 1.1 is not so restrictive. By Plancherel's theorem, it may be stated in terms of the density of $X$ as the property

$$
\int_{-\infty}^{\infty} x^{2} p(x)^{2} d x<\infty
$$

This holds true as long as $p$ is bounded, and $\mathbb{E} X^{2}<\infty$.

As for the condition (1.2), it is of a different nature and is also fulfilled for a certain family of characteristic functions. This family includes, for example, $f(t)=\frac{\sin t}{t}$ which corresponds to the uniform distribution $U$ on the interval $[-1,1]$, and more generally $f(t)=g(t) \frac{\sin t}{t}$ with an arbitrary characteristic function $g$, which means that the distribution of $X$ contains $U$ as a component. The condition (1.2) may also be stated explicitly in terms of the density $p$, by virtue of the Poisson summation formula. As we will see, if $p$ has a bounded total variation, (1.2) is equivalent to the property that

$$
2 \sum_{k \in \mathbb{Z}} \int_{-\infty}^{\infty} p(2 k+x) p(x) d x=1 .
$$

As a relatively large subfamily, one may involve all characteristic functions $f$ that are supported on $[-\pi, \pi]$, in which case we obtain the uniform convergence (1.3). But, staying in a similar class, one may remove the assumption that $X_{n}$ have a Bernoulli distribution and allow a multidimensional setting. In the sequel, we use the standard notations $\langle\cdot, \cdot\rangle$ and $|\cdot|$ to denote respectively the canonical inner product and the Euclidean norm in $\mathbb{R}^{d}$. A random vector $Y=\left(Y_{1}, \ldots, Y_{d}\right)$ in $\mathbb{R}^{d}$ is said to have an isotropic distribution, if

$$
\mathbb{E}\langle Y, \theta\rangle^{2}=|\theta|^{2} \quad \text { for all } \theta \in \mathbb{R}^{d} .
$$

Equivalently, $\mathbb{E} Y_{j} Y_{k}=\delta_{j k}$ for all $j, k \leq d$, where $\delta_{j k}$ is the Kronecker symbol. 
In the next statement, we assume that $X$ is a random vector in $\mathbb{R}^{d}$ with characteristic function $f(t)=\mathbb{E} e^{i\langle t, X\rangle}, t \in \mathbb{R}^{d}$, and that $\left(X_{n}\right)_{n \geq 1}$ are mean zero, independent, identically distributed random vectors in $\mathbb{R}^{d}$ with an isotropic distribution. By the central limit theorem, the normalized sums $Z_{n}$ are convergent weakly in distribution to the standard normal law in $\mathbb{R}^{d}$ with density

$$
\varphi(x)=\frac{1}{(2 \pi)^{d / 2}} e^{-|x|^{2} / 2}, \quad x \in \mathbb{R}^{d} .
$$

Theorem 1.3. There exists $T>0$ depending on the distribution of $X_{1}$ with the following property. If $f$ is supported on the ball $|t| \leq T$, then the random vectors $Z_{n}$ have continuous densities $p_{n}$ such that (1.3) holds true. If

$$
\beta_{3}=\sup _{|\theta|=1} \mathbb{E}\left|\left\langle X_{1}, \theta\right\rangle\right|^{3}
$$

is finite, one may take $T=1 / \beta_{3}$. If $X_{1}$ has a non-lattice distribution, $T$ may be arbitrary.

Theorems 1.1-1.2 also admit multidimensional extensions, which we discuss in Sections 2-3. Theorem 1.3 is proved in Section 4. In Sections 5-6 we recall the Poisson formula, including the multidimensional case, and discuss its applications to (1.2). In the last Section 7, we consider an asymptotic behavior of densities $p_{n}$ in dimension one without the property (1.2). Under mild regularity assumptions on the distribution of $X$, it will be shown in particular that uniformly over all $x$

$$
p_{n}(x)=A_{n}(x) \varphi(x)+O\left(\frac{\log n}{\sqrt{n}}\right), \quad A_{n}(x)=2 \sum_{m \in \mathbb{Z}} p(2 m+x \sqrt{n}+n) .
$$

This asymptotic representation illustrates a strong oscillatory behavior of the densities $p_{n}(x)$ for all points $x \neq 0$, which may actually be different for even $n$ versus odd values of $n$.

\section{Multidimensional variant of Theorem 1.1}

We denote by $L^{r}, r \geq 1$, the space of all (complex-valued) functions $u$ on $\mathbb{R}^{d}$ with finite norm

$$
\|u\|_{r}=\left(\int_{\mathbb{R}^{d}}|u(x)|^{r} d x\right)^{1 / r} .
$$

Turning to the multidimensional variant of Theorem 1.1, suppose that $\left(X_{n}\right)_{n \geq 1}$ are independent random vectors uniformly distributed in the discrete cube $\{-1,1\}^{d}$, so that their components (coordinates) represent independent Bernoulli random variables. Also, let $X$ be a random vector in $\mathbb{R}^{d}$ with characteristic function

$$
f(t)=\mathbb{E} e^{i\langle t, X\rangle}, \quad t \in \mathbb{R}^{d} .
$$

Like the one dimensional case, if $X$ has an absolutely continuous distribution, the normalized sums

$$
Z_{n}=\frac{1}{\sqrt{n}}\left(X+X_{1}+\cdots+X_{n}\right)
$$


have (some) densities $p_{n}$. In addition, the distributions of $Z_{n}$ are convergent weakly as $n \rightarrow \infty$ to the standard normal law in $\mathbb{R}^{d}$ with density $\varphi$ given in (1.4). We would like to strengthen this convergence with respect to the $L^{2}$-distance $\left\|p_{n}-\varphi\right\|_{2}$.

Theorem 2.1. If $Z_{n}$ have densities $p_{n}$ such that

$$
\left\|p_{n}-\varphi\right\|_{2} \rightarrow 0 \text { as } n \rightarrow \infty
$$

then

$$
f(\pi k)=0 \quad \text { for all } k \in \mathbb{Z}^{d}, k \neq 0 .
$$

Conversely, suppose that $\mathbb{E}|X|<\infty$ and

$$
\int_{\mathbb{R}^{d}} \frac{|f(t)|\left|f^{\prime}(t)\right|}{\|t\|^{d-1}} d t<\infty
$$

where $\|t\|$ denotes the distance from the point $t \in \mathbb{R}^{d}$ to the lattice $\pi \mathbb{Z}^{d}$. Then, $Z_{n}$ have densities $p_{n}$, and the $L^{2}$-convergence (2.1) holds true under the condition (2.2).

The moment assumption on $X$ guarantees that the characteristic function $f$ has a continuous derivative (gradient) $f^{\prime}=\nabla f$ with its Euclidean norm $\left|f^{\prime}\right|$, so that (2.3) makes sense. This condition implies that $f$ is in $L^{2}$ as stated in Lemma 2.2 below, hence necessarily $X$ and all $Z_{n}$ have densities. In dimension one, the condition (2.3) is fulfilled as long as $f$ and $f^{\prime}$ are in $L^{2}$ (by Cauchy's inequality). If $d \geq 2,(2.3)$ is a bit more complicated; it is fulfilled when

$$
\sum_{k \in \mathbb{Z}^{d}} \max _{t \in Q_{k}}|f(t)|\left|f^{\prime}(t)\right|<\infty
$$

where $Q_{k}=Q+\pi k, Q=\left[-\frac{\pi}{2}, \frac{\pi}{2}\right]^{d}$. This is true, for example, under the decay assumptions such as

$$
|f(t)| \leq \frac{c}{\left(\left(1+\left|t_{1}\right|\right) \ldots\left(1+\left|t_{d}\right|\right)\right)^{\alpha}}, \quad\left|f^{\prime}(t)\right| \leq \frac{c}{\left(\left(1+\left|t_{1}\right|\right) \ldots\left(1+\left|t_{d}\right|\right)\right)^{\alpha}},
$$

holding for all $t=\left(t_{1}, \ldots, t_{d}\right) \in \mathbb{R}^{d}$ with some constants $\alpha>\frac{1}{2}$ and $c>0$. For instance, this is the case, when $X$ is uniformly distributed in the cube $[-1,1]^{d}$.

Lemma 2.2. If the characteristic function $f$ of the random vector $X$ in $\mathbb{R}^{d}$ with finite first absolute moment satisfies the condition (2.3), and $\sum_{k \in \mathbb{Z}^{d}}|f(\pi k)|^{2}<\infty$, then $X$ has an absolutely continuous distribution with density in $L^{2}$. Moreover, if

$$
\int_{\mathbb{R}^{d}} \frac{\left|f^{\prime}(t)\right|}{\|t\|^{d-1}} d t<\infty
$$

and $\sum_{k \in \mathbb{Z}^{d}}|f(\pi k)|<\infty$, then $X$ has a bounded continuous density.

For the proof of the lemma, as well as of Theorem 2.1 and Theorem 3.1, we partition $\mathbb{R}^{d}$ into the cubes $Q_{k}=Q+\pi k$ introduced above, so that $\|t\|=|t-\pi k|$ for $t \in Q_{k}$. 
Proof. For a given $C^{1}$-smooth function $w$ on $\mathbb{R}^{d}$, consider the functions $w_{k}(t)=w(\pi k+t)$, $k \in \mathbb{Z}^{d}$. Since $w_{k}(t)=w_{k}(0)+\int_{0}^{1}\left\langle w_{k}^{\prime}(\xi t), t\right\rangle d \xi$, we have

$$
\left|w_{k}(t)\right| \leq\left|w_{k}(0)\right|+|t| \int_{0}^{1}\left|w_{k}^{\prime}(\xi t)\right| d \xi
$$

Change of the variable $\xi t=s$ leads to

$$
\begin{aligned}
\int_{Q_{k}}|w(t)| d t-\pi^{d}|w(\pi k)| & \leq \int_{Q} \int_{0}^{1}|t|\left|w_{k}^{\prime}(\xi t)\right| d t d \xi \\
& =\int_{Q}\left|w_{k}^{\prime}(s)\right||s|\left[\int_{\frac{2}{\pi}\|s\|_{\infty}}^{1} \xi^{-d-1} d \xi\right] d s \leq c_{d} \int_{Q} \frac{\left|w_{k}^{\prime}(s)\right|}{|s|^{d-1}} d s
\end{aligned}
$$

with some constant $c_{d}$ depending on $d$ only, where $\|s\|_{\infty}=\max _{k}\left|s_{k}\right|$ for $s=\left(s_{1}, \ldots, s_{d}\right) \in \mathbb{R}^{d}$. It follows that

$$
\int_{\mathbb{R}^{d}}|w(t)| d t \leq \pi^{d} \sum_{k}|w(\pi k)|+c_{d} \int_{\mathbb{R}^{d}} \frac{\left|w^{\prime}(t)\right|}{\|t\|^{d-1}} d t .
$$

For the first claim of the lemma, we apply this inequality with $w(t)=|f(t)|^{2}=f(t) f(-t)$. It is $C^{1}$-smooth and satisfies $\left|w^{\prime}(t)\right| \leq 2|f(t)|\left|f^{\prime}(t)\right|$. Hence, the right-hand side of (2.5) is finite, which means that $f \in L^{2}$. Hence, $X$ has density in $L^{2}$ as well, by the Plancherel theorem. Choosing $w(t)=f(t)$, we obtain that $f$ is integrable, so that the second claim follows from the inverse Fourier formula.

Before turning to the proof of Theorem 2.1, note that the property (2.1) is equivalent to the convergence of the $L^{2}$-norms

$$
\left\|p_{n}\right\|_{2} \rightarrow\|\varphi\|_{2} \text { as } n \rightarrow \infty \text {. }
$$

Indeed, formally the latter is weaker than (2.1). On the other hand, assuming (2.6) and applying the central limit theorem with weak convergence, we have

$$
\left\|p_{n}-\varphi\right\|_{2}^{2}=\left\|p_{n}\right\|_{2}^{2}+\|\varphi\|_{2}^{2}-2 \mathbb{E} \varphi\left(Z_{n}\right) \rightarrow 2\|\varphi\|_{2}^{2}-2 \mathbb{E} \varphi(Z)=0,
$$

where $Z$ is a standard normal random vector in $\mathbb{R}^{d}$.

Now, (2.1) requires that, for all $n$ large enough, the characteristic functions

$$
f_{n}(t)=\mathbb{E} e^{i\left\langle t, Z_{n}\right\rangle}=f\left(\frac{t}{\sqrt{n}}\right) v^{n}\left(\frac{t}{\sqrt{n}}\right)
$$

belong to $L^{2}$, where

$$
v(t)=\cos \left(t_{1}\right) \ldots \cos \left(t_{d}\right) \quad \text { for } t=\left(t_{1}, \ldots, t_{d}\right) \in \mathbb{R}^{d} .
$$

Thus, introducing the characteristic function $g(t)=e^{-|t|^{2} / 2}$ of $Z$ and applying the Plancherel theorem, (2.1) may be restated as the property that

$$
\left\|f_{n}\right\|_{2}^{2} \rightarrow\|g\|_{2}^{2}=\pi^{d / 2} .
$$

Proof of Theorem 2.1.

Necessity part. To explore the latter property, consider the integrals

$$
\left\|f_{n}\right\|_{2}^{2}=\int_{\mathbb{R}^{d}}\left|f_{n}(t)\right|^{2} d t=n^{d / 2} \int_{\mathbb{R}^{d}} w(t) v^{2 n}(t) d t, \quad w(t)=|f(t)|^{2} .
$$


Using the partition of $\mathbb{R}^{d}$ as before and the periodicity of the cosine function, we have

$$
\left\|f_{n}\right\|_{2}^{2}=n^{d / 2} \sum_{k} \int_{Q_{k}} w(t) v^{2 n}(t) d t=n^{d / 2} \sum_{k} I_{n, k},
$$

where

$$
I_{n, k}=\int_{Q} w(\pi k+t) v^{2 n}(t) d t
$$

Given $\varepsilon>0$, choose $t_{0}>0$ small enough such that $w(t) \geq 1-\varepsilon$ in $|t| \leq t_{0}$. We have

$$
I_{n, 0} \geq(1-\varepsilon) \int_{|t| \leq t_{0}} v^{2 n}(t) d t=\frac{1-\varepsilon}{n^{d / 2}} \int_{|t| \leq t_{0} \sqrt{n}} v^{2 n}\left(\frac{t}{\sqrt{n}}\right) d t
$$

implying that

$$
\liminf _{n \rightarrow \infty}\left[n^{d / 2} I_{n, 0}\right] \geq(1-\varepsilon) \int_{\mathbb{R}^{d}} e^{-|t|^{2}} d t=(1-\varepsilon) \pi^{d / 2} .
$$

Since $\varepsilon>0$ was arbitrary, we get

$$
\liminf _{n \rightarrow \infty}\left[n^{d / 2} I_{n, 0}\right] \geq \pi^{d / 2} .
$$

A similar upper bound on limsup is obvious, and we conclude that

$$
n^{d / 2} I_{n, 0} \rightarrow \pi^{d / 2} \text {. }
$$

Now, suppose that (2.2) is violated for some $k \neq 0$, that is, $w(\pi k)>0$. By the continuity of $w$, there exist $\varepsilon>0$ and $t_{0}>0$ such that $w(\pi k+t) \geq \varepsilon$ in $|t| \leq t_{0}$. Hence,

$$
I_{n, k} \geq \varepsilon \int_{|t| \leq t_{0}} v^{2 n}(t) d t=\frac{\varepsilon}{n^{d / 2}} \int_{|t| \leq t_{0} \sqrt{n}} v^{2 n}\left(\frac{t}{\sqrt{n}}\right) d t
$$

implying that

$$
\liminf _{n \rightarrow \infty}\left[n^{d / 2} I_{n, k}\right] \geq \varepsilon \int_{\mathbb{R}^{d}} e^{-|t|^{2}} d t=\varepsilon \pi^{d / 2} .
$$

Combining this bound with (2.9), we eventually obtain in (2.8) that

$$
\liminf _{n \rightarrow \infty}\left\|f_{n}\right\|_{2}^{2} \geq(1+\varepsilon) \pi^{d / 2},
$$

which contradicts to (2.7). This proves the necessity part in Theorem 2.1.

Sufficiency part. By Lemma 2.2, the characteristic functions $f_{n}$ belong to $L^{2}$, so that the densities $p_{n}$ are in $L^{2}$ as well. To prove the required relation (2.7), let us return to the representation (2.8). Recalling (2.9), our task is therefore to show that

$$
n^{d / 2} \sum_{k \neq 0} I_{n, k} \rightarrow 0 \quad(n \rightarrow \infty) .
$$

To this aim, for a fixed $k \neq 0$, using $0 \leq \cos u \leq e^{-u^{2} / 2}$ for $|u| \leq \frac{\pi}{2}$, we have

$$
I_{n, k} \leq J_{n, k}=\int_{Q} w_{k}(t) e^{-n|t|^{2}} d t
$$


where $w_{k}(t)=w(\pi k+t), w(t)=|f(t)|^{2}$ as in the proof of Lemma 2.2. Hence, by (2.4), and changing the variable $\xi t=s$, and then $\xi=\sqrt{n}|s| \frac{1}{u}$, we get

$$
\begin{aligned}
J_{n, k} & \leq \int_{Q} \int_{0}^{1}|t|\left|w_{k}^{\prime}(\xi t)\right| e^{-n|t|^{2}} d t d \xi \\
& \leq \int_{Q}\left|w_{k}^{\prime}(s)\right||s|\left[\int_{0}^{1} \xi^{-d-1} e^{-n|s|^{2} / \xi^{2}} d \xi\right] d s \\
& \leq n^{-d / 2} \int_{Q}\left|w_{k}^{\prime}(s)\right||s|^{-(d-1)}\left[\int_{|s| \sqrt{n}}^{\infty} u^{d-1} e^{-u^{2}} d u\right] d s \\
& \leq c_{d} n^{-d / 2} \int_{Q} \frac{\left|w_{k}^{\prime}(s)\right|}{|s|^{d-1}} e^{-n|s|^{2} / 2} d s
\end{aligned}
$$

with some constant $c_{d}$ depending on the dimension, only. Performing summation over all $k \neq 0$, we get

$$
n^{d / 2} \sum_{k \neq 0} I_{n, k} \leq c_{d} \int_{\|s\|_{\infty}>\frac{\pi}{2}} \frac{\left|w^{\prime}(s)\right|}{\|s\|^{d-1}} e^{-n\|s\|^{2} / 2} d s .
$$

Since $\left|w^{\prime}(s)\right| \leq 2|f(s)|\left|f^{\prime}(s)\right|$, and recalling the assumption (2.3), one may apply the Lebesgue dominated convergence theorem and conclude that the right-hand side of (2.11) tends to zero, and thus (2.7) and (2.10) hold true.

\section{Multidimensional extension of Theorem 1.2}

Keeping notations and the setting of the previous section, the multidimensional variant of Theorem 1.2 reads as follows.

Theorem 3.1. Let $X$ be a random vector in $\mathbb{R}^{d}$ with $\mathbb{E}|X|<\infty$ and with characteristic function $f$ such that

$$
\int_{\mathbb{R}^{d}} \frac{\left|f^{\prime}(t)\right|}{\|t\|^{d-1}} d t<\infty
$$

where $\|t\|$ denotes the distance from $t \in \mathbb{R}^{d}$ to the lattice $\pi \mathbb{Z}^{d}$. If $f(\pi k)=0$ for all $k \in \mathbb{Z}^{d}$, $k \neq 0$, then the normalized sums $Z_{n}$ have continuous densities $p_{n}$ such that

$$
\sup _{x}\left|p_{n}(x)-\varphi(x)\right| \rightarrow 0 \quad \text { as } n \rightarrow \infty \text {. }
$$

In dimension one, (3.1) means that $\left|f^{\prime}\right|$ is integrable. If $d \geq 2$, this condition is fulfilled when

$$
\sum_{k \in \mathbb{Z}^{d}} \max _{t \in Q_{k}}\left|f^{\prime}(t)\right|<\infty
$$

for example, under the decay assumptions such as

$$
\left|f^{\prime}(t)\right| \leq \frac{c}{\left(\left(1+\left|t_{1}\right|\right) \ldots\left(1+\left|t_{d}\right|\right)\right)^{\alpha}}, \quad t=\left(t_{1}, \ldots, t_{d}\right) \in \mathbb{R}^{d}
$$

with some constants $\alpha>1$ and $c>0$. Note that this is not the case, when $X$ is uniformly distributed in the cube $[-1,1]^{d}$. 
This claim is very similar to Theorem 2.1 , and only minor modifications should be done in the proof of the sufficiency part.

Proof. As before, put $v(t)=\cos \left(t_{1}\right) \ldots \cos \left(t_{d}\right)$. By Lemma 2.2, the characteristic functions

$$
f_{n}(t)=f\left(\frac{t}{\sqrt{n}}\right) v^{n}\left(\frac{t}{\sqrt{n}}\right)
$$

are integrable. Hence, $Z_{n}$ have continuous densities given by the Fourier inversion formula

$$
p_{n}(x)=\frac{1}{(2 \pi)^{d}} \int_{\mathbb{R}^{d}} e^{-i\langle t, x\rangle} f_{n}(t) d t=n^{d / 2} \sum_{k \in \mathbb{Z}^{d}} I_{n, k}(x),
$$

where

$$
I_{n, k}(x)=\frac{1}{(2 \pi)^{d}} \int_{Q_{k}} e^{-i\langle t, x\rangle \sqrt{n}} f(t) v^{n}(t) d t
$$

In particular,

$$
n^{d / 2} I_{n, 0}(x)=\frac{1}{(2 \pi)^{d}} \int_{\sqrt{n} Q} e^{-i\langle t, x\rangle} f\left(\frac{t}{\sqrt{n}}\right) v^{n}\left(\frac{t}{\sqrt{n}}\right) d t .
$$

Here, one may remove $f$ from the integrand by using the bound $\left|f\left(\frac{t}{\sqrt{n}}\right)-1\right| \leq \frac{|t|}{\sqrt{n}} \mathbb{E}|X|$. More precisely, this may be done at the expense of an error not exceeding in absolute value

$$
\frac{1}{\sqrt{n}} \mathbb{E}|X| \int_{\sqrt{n} Q}|t| v^{n}\left(\frac{t}{\sqrt{n}}\right) d t \leq \frac{1}{\sqrt{n}} \mathbb{E}|X| \int_{\sqrt{n} Q}|t| e^{-|t|^{2} / 2} d t \leq \frac{c}{\sqrt{n}} \mathbb{E}|X|
$$

up to some absolute constant $c>0$. Hence

$$
n^{d / 2} I_{n, 0}(x)=\frac{1}{(2 \pi)^{d}} \int_{\sqrt{n} Q} e^{-i\langle t, x\rangle} v^{n}\left(\frac{t}{\sqrt{n}}\right) d t+\theta_{n}(x),
$$

where $\sup _{x}\left|\theta_{n}(x)\right| \rightarrow 0$ as $n \rightarrow \infty$. One may now turn to the approximation of $v^{n}$ by the Gaussian function. With some absolute constant $c>0$, we have

$$
\left|\cos ^{n}\left(\frac{u}{\sqrt{n}}\right)-e^{-u^{2} / 2}\right| \leq \frac{c}{n} e^{-u^{2} / 4}, \quad|u| \leq \frac{\pi}{2},
$$

which implies

$$
\left|v^{n}\left(\frac{t}{\sqrt{n}}\right)-e^{-|t|^{2} / 2}\right| \leq \frac{c d}{n} e^{-|t|^{2} / 4}, \quad t=\left(t_{1}, \ldots, t_{d}\right) \in Q .
$$

Therefore, after another replacement, (3.5) is simplified to

$$
n^{d / 2} I_{n, 0}(x)=\frac{1}{(2 \pi)^{d}} \int_{\sqrt{n} Q} e^{-i\langle t, x\rangle} e^{-|t|^{2} / 2} d t+\theta_{n, 1}(x)=\varphi(x)+\theta_{n, 2}(x),
$$

where $\sup _{x}\left|\theta_{n, j}(x)\right| \rightarrow 0$ as $n \rightarrow \infty$. Thus, the term in (3.3) corresponding to $k=0$ produces the desired normal approximation, and we are left to show that $\sum_{k \neq 0}\left|I_{n, k}(x)\right| \rightarrow 0$ as $n \rightarrow \infty$ uniformly over all $x \in \mathbb{R}^{d}$.

For a fixed $k \neq 0$, put $w_{k}(t)=f(\pi k+t)$. Applying again $0 \leq \cos u \leq e^{-u^{2} / 2}$ for $|u| \leq \frac{\pi}{2}$ in (3.4), we have

$$
\left|I_{n, k}(x)\right| \leq J_{n, k}, \quad J_{n, k}=\frac{1}{(2 \pi)^{d}} \int_{Q}\left|w_{k}(t)\right| e^{-n|t|^{2} / 2} d t
$$


Using (2.4), we therefore obtain in full analogy with the derivation from the previous section that

$$
J_{n, k} \leq c_{d} n^{-d / 2} \int_{Q} \frac{\left|w_{k}^{\prime}(s)\right|}{|s|^{d-1}} e^{-n|s|^{2} / 2} d s
$$

with some constant $c_{d}$ depending on the dimension, only. Performing summation over all $k \neq 0$, we get

$$
n^{d / 2} \sum_{k \neq 0}\left|I_{n, k}(x)\right| \leq c_{d} \int_{\|s\|_{\infty}>\frac{\pi}{2}} \frac{\left|f^{\prime}(s)\right|}{\|s\|^{d-1}} e^{-n\|s\|^{2} / 2} d s .
$$

Finally, by (3.1), one may apply the Lebesgue dominated convergence theorem and conclude that the right-hand side of (3.6) tends to zero, and thus (3.2) holds true.

\section{Proof of Theorem $\mathbf{1 . 3}$}

The argument is rather standard, cf. e.g. [P1-2]. Let $v(t)=\mathbb{E} e^{i\left\langle t, X_{1}\right\rangle}, t \in \mathbb{R}^{d}$, be the common characteristic function of $X_{k}$ 's. If $f$ is supported on the ball $|t| \leq T$, the characteristic functions

$$
f_{n}(t)=f\left(\frac{t}{\sqrt{n}}\right) v^{n}\left(\frac{t}{\sqrt{n}}\right)
$$

of the normalized sums $Z_{n}$ are supported on the ball $B_{n}$ of radius $T \sqrt{n}$. Hence, $Z_{n}$ have continuous densities given according to the Fourier inversion formula

$$
p_{n}(x)=\frac{1}{(2 \pi)^{d}} \int_{\mathbb{R}^{d}} e^{-i\langle t, x\rangle} f_{n}(t) d t=\frac{1}{(2 \pi)^{d}} \int_{B_{n}} e^{-i\langle t, x\rangle} f_{n}(t) d t .
$$

In order to explore an asymptotic behavior of these integrals, first note that one may always choose a number $T>0$ such that, for any $0<t_{0}<T$,

$$
c\left(t_{0}\right)=\max _{t_{0} \leq|t| \leq T}|v(t)|<1 .
$$

Moreover, by the second moment assumption,

$$
v(t)=1-\frac{1}{2}|t|^{2}+\varepsilon(t)|t|^{2}
$$

with $\varepsilon(t) \rightarrow 0$ as $t \rightarrow 0$. Let us choose $t_{0} \in(0, T]$ such that $|\varepsilon(t)| \leq \frac{1}{4}$ for all $|t| \leq t_{0}$. Then $|v(t)| \leq 1-\frac{1}{4}|t|^{2}$ in this ball, and

$$
\left|f_{n}(t)\right| \leq\left(1-\frac{1}{4 n}|t|^{2}\right)^{n} \leq e^{-|t|^{2} / 4}, \quad|t| \leq t_{0} \sqrt{n}
$$

Combining this estimate with (4.2), we conclude that for any sequence $T_{n} \uparrow \infty$ with $T_{n} \leq t_{0} \sqrt{n}$,

$$
\int_{T_{n} \leq|t| \leq T \sqrt{n}}\left|f_{n}(t)\right| d t \leq c^{n}(2 T \sqrt{n})^{d} \omega_{d}+\int_{|t| \geq T_{n}} e^{-|t|^{2} / 4} d t \rightarrow 0,
$$

where $c=c\left(t_{0}\right)$, and $\omega_{d}$ denotes the volume of the $d$-dimensional Euclidean unit ball.

Using the principal value of the logarithm, by Taylor expansion, for $|t| \leq t_{0}$ we also have

$$
\log v(t)=\log \left(1-\frac{1}{2}|t|^{2}+\varepsilon(t)|t|^{2}\right)=-\frac{1}{2}|t|^{2}+\varepsilon_{1}(t)|t|^{2}
$$


with $\varepsilon_{1}(t) \rightarrow 0$ as $t \rightarrow 0$. Therefore,

$$
v^{n}\left(\frac{t}{\sqrt{n}}\right)=\exp \left\{-\frac{1}{2}|t|^{2}+\varepsilon\left(\frac{t}{\sqrt{n}}\right)|t|^{2}\right\} \rightarrow g(t)=e^{-|t|^{2} / 2},
$$

where the convergence is uniform in the balls $|t| \leq T_{n}$ such that $T_{n}=o(\sqrt{n})$ as $n \rightarrow \infty$. Hence,

$$
\delta_{n}=\sup _{|t| \leq T_{n}}\left|f_{n}(t)-g(t)\right| \rightarrow 0 .
$$

Moreover, if $T_{n} \uparrow \infty$ sufficiently slow,

$$
\int_{|t| \leq T_{n}}\left|f_{n}(t)-g(t)\right| d t \leq \delta_{n}\left(2 T_{n}\right)^{d} \rightarrow 0
$$

as $n \rightarrow \infty$. Thus, by (4.3),

$$
\begin{aligned}
\int_{Q_{n}}\left|f_{n}(t)-g(t)\right| d t \leq & \int_{|t| \leq T_{n}}\left|f_{n}(t)-g(t)\right| d t \\
& +\int_{T_{n} \leq|t| \leq T \sqrt{n}}\left|f_{n}(t)\right| d t+\int_{T_{n} \leq|t| \leq T \sqrt{n}}|g(t)| d t \rightarrow 0 .
\end{aligned}
$$

In view of (4.1), we obtain the desired relation (1.3), that is,

$$
\left|p_{n}(x)-\varphi(x)\right| \leq \frac{1}{(2 \pi)^{d}} \int_{\mathbb{R}^{d}}\left|f_{n}(t)-g(t)\right| d t \rightarrow 0 .
$$

If $X_{1}$ has a non-lattice distribution, the property (4.2) holds true with any $T>0$, cf. [BR-R], Section 21. Otherwise, let us mention how one may quantify the choice of $T$ satisfying (4.2). If $\xi$ is a mean zero random variable with $\mathbb{E}|\xi|^{3}<\infty$, one has (cf. e.g. [B], Lemma 15.1)

$$
\left|\mathbb{E} e^{i r \xi}\right| \leq \exp \left\{-\frac{r^{2}}{2} \mathbb{E} \xi^{2}+\frac{r^{3}}{3} \mathbb{E}|\xi|^{3}\right\}, \quad r \in \mathbb{R} .
$$

Applying this bound with $\xi=\left\langle X_{1}, \theta\right\rangle, \theta=t /|t|, r=|t|, t \in \mathbb{R}^{d}$, we get

$$
|v(t)| \leq \exp \left\{-\frac{|t|^{2}}{2}+\frac{|t|^{3}}{3} \beta_{3}(\theta)\right\} \leq \exp \left\{-\frac{|t|^{2}}{2}+\frac{|t|^{3}}{3} \beta_{3}\right\}
$$

where $\beta_{3}(\theta)=\mathbb{E}\left|\left\langle X_{1}, \theta\right\rangle\right|^{3}$. If $|t| \leq 1 / \beta_{3}$, the above right-hand side does not exceed $e^{-|t|^{2} / 6}$. Hence, $T=1 / \beta_{3}$ is admissible.

Remark 4.1. One may remove the 3 rd moment assumption and take $T=\pi$ in Theorem 1.3 (in dimension one) under the following hypotheses about the distribution of $X_{1}$ (in addition to the basic moment assumptions $\mathbb{E} X_{1}=0$ and $\left.\mathbb{E} X_{1}^{2}=1\right)$ :

a) The distribution of $X_{1}$ is symmetric about the origin;

b) $\mathbb{P}\left\{X_{1}=0\right\}=0$;

c) The distribution of $X_{1}$ is different than the symmetric Bernoulli distribution on $\{-1,1\}$.

In that case, the property (4.2) still holds true. Indeed, otherwise take the smallest $t_{0}>0$ such that $\left|v\left(t_{0}\right)\right|=1$. This implies that $X_{1}$ has a lattice distribution supported on $a+h \mathbb{Z}$ with $h=2 \pi / t_{0}$ (cf. [P2], Chapter 1, Lemma 3). Equivalently, $X_{1}=a+h \xi$ for some integer-valued random variable $\xi$. By the assumption $a$ ), necessarily $a=h \mathrm{~m} / 2$ for some integer $m$. Adding an integer number to $\xi$, we may assume without loss of generality that $m=0$ or $m=1$. 
In the first case, $X_{1}=h \xi$, so that, by $\left.b\right),\left|X_{1}\right| \geq h$ and thus $1=\mathbb{E} X_{1}^{2} \geq h^{2}$. Hence $t_{0}=\frac{2 \pi}{h} \geq 2 \pi$, implying that (4.2) holds with any $T<2 \pi$. In the second case, $X_{1}=h\left(\frac{1}{2}+\xi\right)$, hence $\left|X_{1}\right| \geq \frac{1}{2} h$ and thus $1=\mathbb{E} X_{1}^{2} \geq h^{2} / 4$. Here, by $a$ ), the equality is only possible when $\xi$ takes the values 0 and 1 with probability $1 / 2$, which is excluded by $c$ ). Hence $h<2$ and $t_{0}=\frac{2 \pi}{h}>\pi$, implying that (4.2) holds with $T=\pi$.

\section{Poisson formula}

As we mentioned before, the property (1.2), needed in Theorems 1.1-1.2 and their multidimensional variants, may be stated explicitly in terms of the density of $X$. Such a reformulation is based on the Poisson formula which we recall in this section.

Consider the Fourier transform

$$
f(t)=\int_{\mathbb{R}^{d}} e^{i\langle t, x\rangle} p(x) d x, \quad t \in \mathbb{R}^{d},
$$

for a given integrable function $p: \mathbb{R}^{d} \rightarrow \mathbb{C}$. The Poisson formula indicates that, under certain mild assumptions on $p$ (or $f$ ), we have the equality

$$
\sum_{m \in \mathbb{Z}^{d}} p(m)=\sum_{k \in \mathbb{Z}^{d}} f(2 \pi k)
$$

In dimension $d=1$, it is sufficient to require that $p$ be continuous and have a bounded total variation on the real line. In this case, the left series in (5.1) is absolutely convergent, while the value of the right series is understood as the limit of the corresponding symmetric sums, cf. [Z], Theorem 13.5. For higher dimensions, (5.1) holds true as long as $p$ belongs to the Schwarz space of functions on $\mathbb{R}^{d}$, as mentioned in [I-K], Theorem 4.5.

Let us recall a standard argument and indicate somewhat weaker conditions in terms of $f$, enlarging the Schwarz class, but restricting ourselves to the case where $p$ or $f$ are real-valued and non-negative.

Proposition 5.1. Let $p$ be an integrable non-negative function on $\mathbb{R}^{d}$ whose Fourier transform $f$ is also integrable and has a continuous derivative $f^{\prime}=\nabla f$ satisfying

$$
\int_{\mathbb{R}^{d}} \frac{\left|f^{\prime}(t)\right|}{\|t\|^{d-1}} d t<\infty
$$

where $\|t\|$ denotes the distance from the point $t$ to the lattice $2 \pi \mathbb{Z}^{d}$. Then we have the equality (5.1), in which the second series is absolutely convergent.

As the next proof shows, the differentiability assumption may slightly be relaxed, assuming that $f$ is locally Lipschitz and using the generalized modulus of the gradient

$$
\left|f^{\prime}(t)\right|=\liminf _{s \rightarrow t} \frac{|f(s)-f(t)|}{|s-t|} .
$$

Note that the function $p$ in Proposition 5.1 is bounded and continuous (which we require below), by the integrability of $f$ and by the inverse Fourier formula which may be written as

$$
p(x / 2 \pi)=\int_{\mathbb{R}^{d}} e^{i\langle t, x\rangle} f(-2 \pi t) d t .
$$


This formula also shows that the role of $p$ and $f$ in (5.1) may be interchanged. In that case, Proposition 5.1 may be restated as follows.

Proposition 5.2. Let $p$ be an integrable, locally Lipschitz function on $\mathbb{R}^{d}$ whose Fourier transform $f$ is integrable and non-negative. Suppose that

$$
\int_{\mathbb{R}^{d}} \frac{\left|p^{\prime}(x)\right|}{\|x\|^{d-1}} d x<\infty
$$

where $\|x\|$ denotes the distance from the point $x$ to the lattice $\mathbb{Z}^{d}$. Then we have the equality (5.1), in which the first series is absolutely convergent.

In dimension $d=1$, the above condition on $p$ just means that $p$ has bounded total variation on the real line, and then we arrive at the usual one-dimensional formulation of (5.1) under an additional assumption that $f$ is non-negative.

Proof of Proposition 5.1. Let us partition $\mathbb{R}^{d}$ into the cubes $Q_{k}=Q+2 \pi k, Q=[-\pi, \pi]^{d}$, $k \in \mathbb{Z}^{d}$, and apply the bound

$$
|f(2 \pi k+t)-f(2 \pi k)| \leq|t| \int_{0}^{1}\left|f^{\prime}(2 \pi k+\xi t)\right| d \xi, \quad t \in \mathbb{R}^{d} .
$$

It holds true as long as $f$ is locally Lipschitz, with definition (5.2) of the modulus of the gradient of $f$. Indeed, for any $x, t \in \mathbb{R}^{d}$, the function $u(\xi)=f(x+\xi t)-f(x)$ is locally Lipschitz on the line, and therefore it is absolutely continuous. If $u^{\prime}$ is a Radon-Nikodym derivative of $u$, it follows from (5.2) that $\left|u^{\prime}(\xi)\right| \leq|t|\left|f^{\prime}(x+\xi t)\right|$ a.e., while $|u(1)| \leq \int_{0}^{1}\left|u^{\prime}(\xi)\right| d \xi$.

Now, arguing as in the proof of Lemma 2.2, we have

$$
\begin{aligned}
\int_{Q}|f(2 \pi k+t)-f(2 \pi k)| d t & \leq \int_{0}^{1} \int_{Q}\left|f^{\prime}(2 \pi k+\xi t)\right||t| d \xi d t \\
& =\int_{Q}\left[\left|f^{\prime}(2 \pi k+s)\right||s| \int_{\frac{\|s\|_{\infty}}{\pi}}^{1} \frac{d \xi}{\xi^{d+1}}\right] d s \leq c_{d} \int_{Q} \frac{\left|f^{\prime}(2 \pi k+s)\right|}{|s|^{d-1}} d s
\end{aligned}
$$

with some constant $c_{d}$ depending on $d$ only. Hence

$$
(2 \pi)^{d}|f(2 \pi k)| \leq \int_{Q_{k}}|f(t)| d t+c_{d} \int_{Q_{k}} \frac{\left|f^{\prime}(t)\right|}{\|t\|^{d-1}} d t .
$$

The next summation over all $k$ leads to

$$
\sum_{k \in \mathbb{Z}^{d}}|f(2 \pi k)| \leq \frac{1}{(2 \pi)^{d}} \int_{\mathbb{R}^{d}}|f(t)| d t+\frac{c_{d}}{(2 \pi)^{d}} \int_{\mathbb{R}^{d}} \frac{\left|f^{\prime}(t)\right|}{\|t\|^{d-1}} d t<\infty,
$$

so that the second series in (5.1) is absolutely convergent.

Next, consider the periodic function

$$
P(x)=\sum_{m \in \mathbb{Z}^{d}} p(m+x), \quad x \in \mathbb{R}^{d} .
$$


It is a.e. finite and integrable on the unit cube $K=[0,1]^{d}$, since

$$
\int_{K} \sum_{m \in \mathbb{Z}^{d}} p(m+x) d x=\int_{\mathbb{R}^{d}} p(x) d x<\infty .
$$

Therefore, $P$ admits a multiple Fourier series expansion $\sum_{k \in \mathbb{Z}^{d}} a_{k} e^{-2 \pi i\langle k, x\rangle}$ with coefficients

$$
\begin{aligned}
a_{k} & =\int_{K} e^{2 \pi i\langle k, x\rangle} P(x) d x=\sum_{m \in \mathbb{Z}^{d}} \int_{K} e^{2 \pi i\langle k, x\rangle} p(x+m) d x \\
& =\sum_{m \in \mathbb{Z}^{d}} \int_{K+m} e^{2 \pi i\langle k, y\rangle} p(y) d y=\int_{\mathbb{R}^{d}} e^{2 \pi i\langle k, y\rangle} p(y) d y=f(2 \pi k) .
\end{aligned}
$$

The Fourier series is thus absolutely convergent, and as a consequence, $P(x)=\tilde{P}(x)$ a.e., where

$$
\tilde{P}(x)=\sum_{k \in \mathbb{Z}^{d}} f(2 \pi k) e^{-2 \pi i\langle k, x\rangle} .
$$

By (5.4), $\tilde{P}$ represents a continuous function. Once $P$ is finite and continuous as well, we could conclude that $P(x)=\tilde{P}(x)$ for all $x \in \mathbb{R}^{d}$. But, for $x=0$, the latter equality becomes the Poisson formula (5.1).

The boundedness and continuity of $P$ (needed at zero only) may be explored in terms of smoothness properties of $p$. Instead, let us apply a smoothing argument. Using the Fourier couple on the real line,

$$
w(x)=\left(\frac{\sin (\pi x)}{\pi x}\right)^{2}, \quad \hat{w}(t)=\left(1-\frac{|t|}{2 \pi}\right)^{+},
$$

the function $w_{T}(x)=w(x / T)$ with a parameter $T \geq 1$ has the Fourier transform $\hat{w}_{T}(t)=$ $T \hat{w}(T t), x, t \in \mathbb{R}$. Define

$$
w_{T}(x)=w_{T}\left(x_{1}\right) \ldots w_{T}\left(x_{d}\right), \quad x=\left(x_{1}, \ldots, x_{d}\right) \in \mathbb{R}^{d},
$$

with its Fourier transform

$$
\hat{w}_{T}(t)=\hat{w}_{T}\left(t_{1}\right) \ldots \hat{w}_{T}\left(t_{d}\right), \quad t=\left(t_{1}, \ldots, t_{d}\right) \in \mathbb{R}^{d} .
$$

Put $p_{T}(x)=p(x) w_{T}(x)$ with the corresponding periodic function

$$
P_{T}(x)=\sum_{m \in \mathbb{Z}^{d}} p(m+x) w_{T}(m+x) .
$$

Since $p$ is bounded, the above series is absolutely convergent. Indeed, using $|w(x / T)| \leq \frac{c T^{2}}{1+x^{2}}$, $x \in \mathbb{R}$, we have, for any $x=\left(x_{1}, \ldots, x_{d}\right) \in \mathbb{R}^{d}$ with $\|x\|_{\infty} \leq 1$,

$$
\begin{aligned}
w_{T}(m+x) & \leq \frac{\left(c T^{2}\right)^{d}}{\left(1+\left(m_{1}+x_{1}\right)^{2}\right) \ldots\left(1+\left(m_{d}+x_{d}\right)^{2}\right)} \\
& \leq \frac{\left(c T^{2}\right)^{d}}{\left(1+m_{1}^{2}\right) \ldots\left(1+m_{d}^{2}\right)}, \quad m=\left(m_{1}, \ldots, m_{d}\right) \in \mathbb{Z}^{d},
\end{aligned}
$$

where $c$ denotes an absolute constant which may be different in different places. It follows that the sum of the series in (5.5) is uniformly bounded. Since all terms in (5.5) are continuous in $x$, we may conclude that $P_{T}$ is continuous as well. 
It also follows from (5.5) and the property $w_{T}(0)=1$ that

$$
\lim _{T \rightarrow \infty} P_{T}(0)=P(0) \text {. }
$$

It is the only place where the property that $p$ is non-negative is used. Since $0 \leq w_{T} \leq 1$, we have $\lim \sup _{T \rightarrow \infty} P_{T}(0) \leq P(0)$. On the other hand, since $w_{T}(x) \rightarrow 1$, for any fixed $N \geq 1$

$$
\liminf _{T \rightarrow \infty} P_{T}(0) \geq \liminf _{T \rightarrow \infty} \sum_{\|m\|_{\infty} \leq N} p(m) w_{T}(m)=\sum_{\|m\|_{\infty} \leq N} p(m) .
$$

Since $N$ is arbitrary, we get $\liminf _{T \rightarrow \infty} P_{T}(0) \geq P(0)$ and thus arrive at (5.6).

Now, the Fourier transform $f_{T}$ of $p_{T}$ represents the normalized convolution $(2 \pi)^{-d} f * \hat{w}_{T}$, which is integrable and satisfies

$$
\int_{\mathbb{R}^{d}} \frac{\left|f_{T}^{\prime}(t)\right|}{\|t\|^{d-1}} d t<\infty
$$

The latter follows from the equality $f_{T}^{\prime}=(2 \pi)^{-d} f^{\prime} * \hat{w}_{T}=(2 \pi)^{-d} f * \hat{w}_{T}^{\prime}$ together with the bound $\int_{\mathbb{R}^{d}} \frac{\left|w_{T}^{\prime}(t)\right|}{\|t-s\|^{d-1}} d t \leq C(T)$ holding true with a constant $C(T)$ independent of $s$. Thus,

$$
\sum_{k \in \mathbb{Z}^{d}}\left|f_{T}(2 \pi k)\right|<\infty
$$

and we obtain the Poisson formula for the smoothed functions, that is,

$$
P_{T}(0)=\tilde{P}_{T}(0) \equiv \sum_{k \in \mathbb{Z}^{d}} f_{T}(2 \pi k) .
$$

In order to turn to the limit in this equality, note that $(2 \pi)^{-d} \int_{\mathbb{R}^{d}} \hat{w}_{T}(t) d t=w_{T}(0)=$ $w(0)=1$, so that we may write

$$
f_{T}(2 \pi k)-f(2 \pi k)=(2 \pi)^{-d} \int_{\mathbb{R}^{d}}(f(2 \pi k+t)-f(2 \pi k)) \hat{w}_{T}(t) d t .
$$

Hence, by (5.3),

$$
\left|f_{T}(2 \pi k)-f(2 \pi k)\right| \leq(2 \pi)^{-d} \int_{0}^{1} \int_{\mathbb{R}^{d}}\left|f^{\prime}(2 \pi k+\xi t)\right||t| \hat{w}_{T}(t) d \xi d t .
$$

Changing the variable $\xi t=s$ and using $\hat{w}_{T}(t)=0$ for $\|t\|_{\infty} \geq 2 \pi / T$, with $\left|\hat{w}_{T}(t)\right| \leq(c T)^{d}$ for $\|t\|_{\infty} \leq 2 \pi / T$, the last double integral may be bounded by

$$
(c T)^{d} \int_{\|s\|_{\infty} \leq \frac{2 \pi}{T}}\left[\left|f^{\prime}(2 \pi k+s)\right||s| \int_{T\|s\|_{\infty}}^{1} \frac{d \xi}{\xi^{d+1}}\right] d s \leq c_{d} \int_{\|s\|_{\infty} \leq \frac{2 \pi}{T}} \frac{\left|f^{\prime}(2 \pi k+s)\right|}{|s|^{d-1}} d s
$$

with some constant $c_{d}$ depending on $d$ only. Hence, summing over all $k$, we get

$$
\sum_{k \in \mathbb{Z}^{d}}\left|f_{T}(2 \pi k)-f(2 \pi k)\right| \leq c_{d}(2 \pi)^{-d} \int_{R_{T}} \frac{\left|f^{\prime}(t)\right|}{\|t\|^{d-1}} d t,
$$

where $R_{T}=\bigcup_{k}\left(\left[-\frac{2 \pi}{T}, \frac{2 \pi}{T}\right]^{d}+2 \pi k\right)$. This region shrinks to the lattice $2 \pi \mathbb{Z}^{d}$ for growing $T$, while the integral on the right is finite, when the integration is performed over the whole space. Therefore, by the Lebesgue dominated convergence theorem, both sides of (5.8) tend to zero. In particular, $\tilde{P}_{T}(0) \rightarrow \tilde{P}(0)$ as $T \rightarrow \infty$. Thus, in the limit (5.7) together with (5.6) yield the desired equality $\tilde{P}(0)=P(0)$. 


\section{Poisson formula for convoluted densities}

Let us restate once more Propositions 5.1-5.2, assuming that $f$ is the characteristic function of a random vector $X$ in $\mathbb{R}^{d}$.

Proposition 6.1. Let $\mathbb{E}|X|<\infty$, and assume that $f$ is integrable and satisfies

$$
\int_{\mathbb{R}^{d}} \frac{\left|f^{\prime}(t)\right|}{\|t\|^{d-1}} d t<\infty
$$

where $\|t\|$ denotes the distance from $t$ to the lattice $2 \pi \mathbb{Z}^{d}$. Then $X$ has a bounded continuous density $p$, and we have the equality (5.1), in which the second series is absolutely convergent.

Here, the moment assumption on $X$ ensures that $f$ has a continuous derivative $f^{\prime}$.

Proposition 6.2. Let $f$ be integrable and non-negative, and assume that the density $p$ of $X$ is locally Lipschitz and satisfies

$$
\int_{\mathbb{R}^{d}} \frac{\left|p^{\prime}(x)\right|}{\|x\|^{d-1}} d x<\infty
$$

where $\|x\|$ denotes the distance from $x$ to the lattice $\mathbb{Z}^{d}$. Then we have the equality (5.1), in which the sums of both series are finite.

By the integrability of $f$, the random vector $X$ has a bounded continuous density $p$ given by the inverse Fourier formula. It implies in particular that $p$ has a bounded continuous derivative $p^{\prime}$ as soon as $\int_{\mathbb{R}^{d}}|t|\left|f^{\prime}(t)\right| d t<\infty$. The latter condition is however not necessary.

Recall that in dimension $d=1$, the assumptions in Proposition 6.2 may be weakened. It is sufficient to require that $X$ have a continuous density of bounded total variation (removing any hypotheses on $f$ ). This requirement may be related to the properties of the characteristic function. For example, it is sufficient to have (cf. e.g. [B-C-G], Proposition 5.2) that

$$
\int_{-\infty}^{\infty} t^{2}\left(|f(t)|^{2}+\left|f^{\prime}(t)\right|^{2}\right) d t<\infty
$$

In case $d \geq 2$, the assumptions (6.1) and (6.2) are respectively fulfilled under decay bounds

$$
\left|f^{\prime}(t)\right| \leq \frac{c}{\left(\left(1+\left|t_{1}\right|\right) \ldots\left(1+\left|t_{d}\right|\right)\right)^{\alpha}}, \quad\left|p^{\prime}(x)\right| \leq \frac{c}{\left(\left(1+\left|x_{1}\right|\right) \ldots\left(1+\left|x_{d}\right|\right)\right)^{\alpha}},
$$

holding for all $t=\left(t_{1}, \ldots, t_{d}\right) \in \mathbb{R}^{d}$ and respectively $x=\left(x_{1}, \ldots, x_{d}\right) \in \mathbb{R}^{d}$ with some constants $\alpha>1$ and $c>0$. These bounds may be strengthened to

$$
\left|f^{\prime}(t)\right| \leq \frac{c}{(1+|t|)^{\alpha d}}, \quad\left|p^{\prime}(x)\right| \leq \frac{c}{(1+|x|)^{\alpha d}} .
$$

The latter is fulfilled for all functions on $\mathbb{R}^{d}$ from the Schwarz space.

Let us now turn to the density description of the condition $f(\pi k)=0$ for all $k \neq 0$ appearing in Theorems 1.1-1.2 and 2.1-3.1. It may equivalently be stated as the property

$$
\sum_{k \in \mathbb{Z}^{d}}|f(\pi k)|^{2}=1
$$


Note that $v(t)=|f(t / 2)|^{2}$ is non-negative and represents the characteristic function of the random vector $Y=\left(X-X^{\prime}\right) / 2$, where $X^{\prime}$ is an independent copy of $X$. If $X$ has density $p$, the density of $Y$ is given by

$$
q(x)=2^{d} \int_{\mathbb{R}^{d}} p(2 x+y) p(y) d y .
$$

Hence, under the corresponding regularity assumptions, the Poisson formula (5.1) for the couple $(q, v)$ becomes

$$
\sum_{k \in \mathbb{Z}^{d}}|f(\pi k)|^{2}=\sum_{k \in \mathbb{Z}^{d}} q(k)=2^{d} \sum_{k \in \mathbb{Z}^{d}} \int_{\mathbb{R}^{d}} p(2 k+x) p(x) d x,
$$

which is equivalent to (6.3), if and only if

$$
\sum_{k \in \mathbb{Z}^{d}} \int_{\mathbb{R}^{d}} p(2 k+x) p(x) d x=2^{-d} .
$$

Let us precise the regularity assumptions. Since $\left|v^{\prime}(t)\right| \leq 2|f(t)|\left|f^{\prime}(t)\right|$, the condition (6.1) is fulfilled as long as

$$
\int_{\mathbb{R}^{d}} \frac{|f(t)|\left|f^{\prime}(t)\right|}{\|t\|^{d-1}} d t<\infty
$$

where $\|t\|$ denotes the distance from $t$ to the lattice $2 \pi \mathbb{Z}^{d}$. Hence, from Proposition 6.1 we obtain:

Corollary 6.3. Let $\mathbb{E}|X|<\infty$, and assume that $f$ is square integrable and satisfies the condition (6.5). Then $f(\pi k)=0$ for all $k \in \mathbb{Z}^{d}, k \neq 0$, if and only if the equality (6.4) holds.

The assumption that $f \in L^{2}$ implies that $X$ has a square integrable density $p$, in which case the density $q$ is continuous. Let us also note that the condition (6.5) is exactly the assumption (2.3) from Theorem 2.1. Hence, under (6.5), (6.4) is equivalent to the local limit theorem (2.1), that is, to the property

$$
\left\|p_{n}-\varphi\right\|_{2} \rightarrow 0 \quad \text { as } n \rightarrow \infty .
$$

One may also develop an application of Proposition 6.2 to the density $q$ (in place of $p$ ). Assuming that the density $p$ has a continuous derivative, we have that $q$ has the derivative

$$
q^{\prime}(x)=2^{d+1} \int_{\mathbb{R}^{d}} p^{\prime}(2 x+y) p(y) d y .
$$

To weaken the assumptions, consider the one-dimensional case. Then, the only requirement we need to meet is that $q$ is continuous and has a bounded total variation on the real line. The continuity is met as long as $p \in L^{2}$, while $\|q\|_{\mathrm{TV}} \leq 2\|p\|_{\mathrm{TV}}$. Hence, we arrive at:

Corollary 6.4. Assume that the random variable $X$ has a density $p$ with bounded total variation. Then $f(\pi k)=0$ for all $k \in \mathbb{Z}, k \neq 0$, if and only if

$$
\sum_{k \in \mathbb{Z}} \int_{-\infty}^{\infty} p(2 k+x) p(x) d x=\frac{1}{2}
$$




\section{Asymptotic behavior of densities without condition (1.2)}

Let us now return to the setting of Theorem 1.2, thus restricting ourselves to dimension $d=1$. Without the condition (1.2), the densities $p_{n}(x)$ of the normalized sums $Z_{n}$ have an oscillating character at all points $x \neq 0$. Here we describe a typical situation, assuming that the density $p$ of the random variable $X$ is sufficiently regular.

Theorem 7.1. Assume that $X$ has a continuous density $p$ of bounded total variation, with finite second moment. If the characteristic function $f=\mathbb{E} e^{i t X}$ and its derivatives $f^{\prime}$ and $f^{\prime \prime}$ are integrable, then $Z_{n}$ have uniformly bounded densities $p_{n}$ satisfying uniformly over all $x$

$$
p_{n}(x)=A_{n}(x) \varphi(x)+O\left(\frac{\log n}{\sqrt{n}}\right)
$$

where

$$
A_{n}(x)=\sum_{k \in \mathbb{Z}} e^{-i \pi k(x \sqrt{n}+n)} f(\pi k)=2 \sum_{m \in \mathbb{Z}} p(2 m+x \sqrt{n}+n) .
$$

Thus, the behavior of $p_{n}$ might be different for $n$ even and $n$ odd. The point $x=0$ turns out to be special, since then the oscillatory character disappears along even and odd values of $n$ respectively.

Corollary 7.2. Under the same assumptions,

$$
\begin{aligned}
\lim _{n \rightarrow \infty} p_{2 n}(0) & =\frac{2}{\sqrt{2 \pi}} \sum_{m \in \mathbb{Z}} p(2 m), \\
\lim _{n \rightarrow \infty} p_{2 n+1}(0) & =\frac{2}{\sqrt{2 \pi}} \sum_{m \in \mathbb{Z}} p(2 m+1) .
\end{aligned}
$$

Proof of Theorem 7.1. Since $f$ is integrable, the random variables $Z_{n}$ have bounded continuous densities described by the inverse Fourier formula

$$
p_{n}(x)=\frac{1}{2 \pi} \int_{-\infty}^{\infty} e^{-i t x} f_{n}(t) d t, \quad x \in \mathbb{R}
$$

where

$$
f_{n}(t)=f\left(\frac{t}{\sqrt{n}}\right) \cos ^{n}\left(\frac{t}{\sqrt{n}}\right)
$$

are the characteristic functions of $Z_{n}$. As before, let us split the integration in (7.2) into the intervals $\frac{1}{\sqrt{n}} Q_{k}, Q_{k}=\left[\pi k-\frac{\pi}{2}, \pi k+\frac{\pi}{2}\right], k \in \mathbb{Z}$, to get the representation

$$
p_{n}(x)=\frac{1}{2 \pi} \sqrt{n} \sum_{k}(-1)^{n k} e^{-i \pi k x \sqrt{n}} I_{n, k}(x),
$$


with

$$
\begin{aligned}
I_{n, k}(x)= & \int_{-\frac{\pi}{2}}^{\frac{\pi}{2}} e^{-i t x \sqrt{n}} f(\pi k+t) \cos ^{n}(t) d t \\
= & f(\pi k) \int_{-\frac{\pi}{2}}^{\frac{\pi}{2}} e^{-i t x \sqrt{n}} \cos ^{n}(t) d t \\
& +\int_{-\frac{\pi}{2}}^{\frac{\pi}{2}} e^{-i t x \sqrt{n}}(f(\pi k+t)-f(\pi k)) \cos ^{n}(t) d t .
\end{aligned}
$$

Using $\theta, \theta_{j}$ to denote quantities bounded by an absolute constant, from the asymptotic expression

$$
\cos ^{n}(t)=e^{-n t^{2} / 2}+\frac{\theta}{n} e^{-t^{2} / 4}, \quad|t| \leq \frac{\pi}{2}
$$

we obtain that

$$
\begin{aligned}
\int_{-\frac{\pi}{2}}^{\frac{\pi}{2}} e^{-i t x \sqrt{n}} \cos ^{n}(t) d t & =\int_{-\frac{\pi}{2}}^{\frac{\pi}{2}} e^{-i t x \sqrt{n}} e^{-n t^{2} / 2} d t+\frac{\theta_{1}}{n} \\
& =\int_{-\infty}^{\infty} e^{-i t x \sqrt{n}} e^{-n t^{2} / 2} d t+\frac{\theta_{2}}{n}=\frac{\sqrt{2 \pi}}{\sqrt{n}} e^{-x^{2} / 2}+\frac{\theta_{2}}{n}
\end{aligned}
$$

This gives an asymptotic representation for the first integral in (7.4).

The second integral has a smaller order. Put $\varepsilon_{n}=\frac{\sqrt{2 \log n}}{\sqrt{n}}$ (assuming that $\varepsilon_{n} \leq \frac{\pi}{2}$ ). We use $0 \leq \cos t \leq e^{-t^{2} / 2},|t| \leq \frac{\pi}{2}$, so that $\cos ^{n} t \leq \frac{1}{n}$ for $\varepsilon_{n}<|t|<\frac{\pi}{2}$. This implies that

$$
\begin{aligned}
\int_{\varepsilon_{n}}^{\frac{\pi}{2}}|f(\pi k+t)-f(\pi k)| \cos ^{n}(t) d t & \leq \int_{\varepsilon_{n}}^{\frac{\pi}{2}}\left[\int_{0}^{t}\left|f^{\prime}(\pi k+s)\right| \cos ^{n}(t) d s\right] d t \\
& \leq \int_{\varepsilon_{n}}^{\frac{\pi}{2}}\left[\int_{0}^{t}\left|f^{\prime}(\pi k+s)\right| e^{-n t^{2} / 2} d s\right] d t \\
& \leq \frac{2}{n} \int_{0}^{\frac{\pi}{2}}\left|f^{\prime}(\pi k+s)\right| d s .
\end{aligned}
$$

With a similar bound for the interval $-\frac{\pi}{2}<t<-\varepsilon_{n}$, we get

$$
\int_{\varepsilon_{n}<|t|<\frac{\pi}{2}}|f(\pi k+t)-f(\pi k)| \cos ^{n}(t) d t \leq \frac{2}{n} \int_{-\frac{\pi}{2}}^{\frac{\pi}{2}}\left|f^{\prime}(\pi k+s)\right| d s .
$$

For the interval $|t|<\varepsilon_{n}$, we use the Taylor integral formula up to the quadratic form,

$$
f(\pi k+t)-f(\pi k)=f^{\prime}(\pi k) t+\int_{0}^{t}(t-s) f^{\prime \prime}(\pi k+s) d s .
$$

By (7.5), the linear term makes a contribution

$$
\begin{aligned}
\int_{-\varepsilon_{n}}^{\varepsilon_{n}} e^{-i t x \sqrt{n}} t \cos ^{n}(t) d t & =\int_{-\varepsilon_{n}}^{\varepsilon_{n}} e^{-i t x \sqrt{n}} t e^{-n t^{2} / 2} d t+\frac{\theta_{3}}{n} \\
& =\frac{1}{n} \int_{-\varepsilon_{n} \sqrt{n}}^{\varepsilon_{n} \sqrt{n}} e^{-i s x} s e^{-s^{2} / 2} d s+\frac{\theta_{3}}{n}=\frac{\theta_{4}}{n} .
\end{aligned}
$$


Hence, for the integral

we get

$$
J_{n, k}(x)=\int_{|t|<\varepsilon_{n}} e^{-i t x \sqrt{n}}(f(\pi k+t)-f(\pi k)) \cos ^{n}(t) d t,
$$

$$
\begin{aligned}
\left|J_{n, k}(x)\right| & \leq \frac{\theta_{4}}{n}\left|f^{\prime}(\pi k)\right|+\left|\int_{-\varepsilon_{n}}^{\varepsilon_{n}} e^{-i t x \sqrt{n}} \int_{0}^{t}(t-s) f^{\prime \prime}(\pi k+s) \cos ^{n}(t) d t d s\right| \\
& \leq \frac{\theta_{4}}{n}\left|f^{\prime}(\pi k)\right|+\int_{-\varepsilon_{n}}^{\varepsilon_{n}} \int_{-|t|}^{|t|}(|t|-|s|)\left|f^{\prime \prime}(\pi k+s)\right| d t d s \\
& \leq \frac{\theta_{4}}{n}\left|f^{\prime}(\pi k)\right|+\int_{-\varepsilon_{n}}^{\varepsilon_{n}}\left(\varepsilon_{n}-|s|\right)^{2}\left|f^{\prime \prime}(\pi k+s)\right| d s \\
& \leq \frac{\theta_{4}}{n}\left|f^{\prime}(\pi k)\right|+\frac{2 \log n}{n} \int_{-\frac{\pi}{2}}^{\frac{\pi}{2}}\left|f^{\prime \prime}(\pi k+s)\right| d s .
\end{aligned}
$$

Together with (7.6)-(7.7), we thus arrive at

$$
\begin{aligned}
\sqrt{n} I_{n, k}(x)= & \left(\sqrt{2 \pi} e^{-x^{2} / 2}+\frac{\theta_{j}}{\sqrt{n}}\left|f^{\prime}(\pi k)\right|\right) f(\pi k) \\
& +\tilde{\theta}_{j} \frac{\log n}{\sqrt{n}} \int_{-\frac{\pi}{2}}^{\frac{\pi}{2}}\left(\left|f^{\prime}(\pi k+s)\right|+\left|f^{\prime \prime}(\pi k+s)\right|\right) d s
\end{aligned}
$$

with bounded quantities $\theta_{j}$ and $\tilde{\theta}_{j}$.

To perform summation over all $k \in \mathbb{Z}$, first note that $\sum_{k}|f(\pi k)|<\infty$, as was emphasized in Proposition 6.1. Similarly, $\sum_{k}\left|f^{\prime}(\pi k)\right|<\infty$, since $f^{\prime \prime}$ is integrable. Returning to (7.3), we thus obtain that

$$
\begin{aligned}
p_{n}(x)= & (\varphi(x)+O(1 / \sqrt{n})) \sum_{k}(-1)^{n k} e^{-i \pi k x \sqrt{n}} f(\pi k) \\
& +\theta \frac{\log n}{\sqrt{n}} \int_{-\infty}^{\infty}\left(\left|f^{\prime}(s)\right|+\left|f^{\prime \prime}(s)\right|\right) d s,
\end{aligned}
$$

that is, uniformly over all $x$

$$
p_{n}(x)=A_{n}(x) \varphi(x)+O\left(\frac{\log n}{\sqrt{n}}\right), \quad A_{n}(x)=\sum_{k \in \mathbb{Z}} e^{-i \pi k(x \sqrt{n}+n)} f(\pi k) .
$$

Since the factors $A_{n}$ are uniformly bounded, so are $p_{n}$. We now apply Proposition 6.1 to the random variables

$$
\xi_{n}=\frac{1}{2}(X-x \sqrt{n}-n),
$$

whose characteristic functions and densities are given by

$$
v_{n}(t)=\mathbb{E} e^{i t \xi_{n}}=e^{-i t(x \sqrt{n}+n) / 2} f(t / 2), \quad q_{n}(y)=2 p(2 y+x \sqrt{n}+n) .
$$

With this choice we get

$$
A_{n}(x)=\sum_{k \in \mathbb{Z}} v_{n}(2 \pi k)=\sum_{m \in \mathbb{Z}} q_{n}(m)=2 \sum_{m \in \mathbb{Z}} p(2 m+x \sqrt{n}+n) .
$$


This observation implies that we cannot hope to obtain the convergence of $p_{n}$ to $\varphi$ even in $L^{1}$. For example, let us consider the two-sided exponential distribution with density $p(x)=$ $\frac{1}{2} e^{-|x|}$. In this case, by Corollary 7.2 ,

$$
\begin{aligned}
\lim _{n \rightarrow \infty} p_{2 n}(0) & =\frac{1}{\sqrt{2 \pi}} \sum_{m} e^{-2|m|}=\frac{1}{\sqrt{2 \pi}} \frac{e^{2}+1}{e^{2}-1}>\varphi(0), \\
\lim _{n \rightarrow \infty} p_{2 n+1}(0) & =\frac{1}{\sqrt{2 \pi}} \sum_{m} e^{-|2 m+1|}=\frac{1}{\sqrt{2 \pi}} \frac{2 e}{e^{2}-1}<\varphi(0) .
\end{aligned}
$$

The same expressions are obtained for the values $x=2 k / \sqrt{n}$. The function $A_{n}(x)$ has period $2 / \sqrt{n}$. Let $x \sqrt{n}=2 k+h, k \in \mathbb{Z}, 0<h<2$. Then along even indexes $n$,

$$
A_{n}(x)=2 \sum_{m \in \mathbb{Z}} p(2 m+h)=\frac{e^{h}+e^{2-h}}{e^{2}-1} .
$$

The latter expression is bounded away from zero for all $h$ small enough. Hence, according to (7.1), we have $\int_{-\infty}^{\infty}\left|p_{n}(x)-\varphi(x)\right| d x \geq c>0$.

\section{REFERENCES}

[B-RR] Bhattacharya, R. N.; Ranga Rao, R. Normal approximation and asymptotic expansions. John Wiley \& Sons, Inc. 1976. Also: Soc. for Industrial and Appl. Math., Philadelphia, 2010.

[B] Bobkov, S. G. Asymptotic expansions for products of characteristic functions under moment assumptions of non-integer orders. Convexity and concentration, 297-357, IMA Vol. Math. Appl., 161, Springer, New York, 2017.

[B-C-G] Bobkov, S. G.; Chistyakov, G. P.; Götze, F. Fisher information and the central limit theorem. Probab. Theory Related Fields 159 (2014), issue 1-2, 1-59.

[G-K] Gnedenko, B. V.; Kolmogorov, A. N. Limit distributions for sums of independent random variables. Translated and annotated by K. L. Chung. With an Appendix by J. L. Doob. AddisonWesley Publishing Company, Inc., Cambridge, Mass., 1954. ix+264 pp.

[I-K] Iwaniec, H.; Kowalski, E. Analytic number theory. American Mathematical Society Colloquium Publications, 53. American Mathematical Society, Providence, RI, 2004. xii+615.

[P1] Petrov, V. V. Local limit theorems for sums of independent random variables. (Russian) Teor. Verojatnost. Primenen. 9 (1964), 343-352.

[P2] Petrov, V. V. Sums of independent random variables. Translated from the Russian by A. A. Brown. Ergebnisse der Mathematik und ihrer Grenzgebiete, Band 82. Springer-Verlag, New YorkHeidelberg, 1975. x+346 pp. Russian ed.: Moscow, Nauka, 1972, 414 pp.

[Z] Zygmund, A. Trigonometric series. 2nd ed. Vol. I. Cambridge University Press, New York 1959, xii+383 pp. 